\title{
Interindividual variability and intraindividual reliability of intermittent theta burst stimulation-induced neuroplasticity mechanisms in the healthy brain
}

Citation for published version (APA):

Schilberg, L., Schuhmann, T., \& Sack, A. T. (2017). Interindividual variability and intraindividual reliability of intermittent theta burst stimulation-induced neuroplasticity mechanisms in the healthy brain. Journal of Cognitive Neuroscience, 29(6), 1022-1032. https://doi.org/10.1162/jocn_a_01100

Document status and date:

Published: 01/06/2017

DOI:

10.1162/jocn_a_01100

Document Version:

Publisher's PDF, also known as Version of record

Document license:

Taverne

Please check the document version of this publication:

- A submitted manuscript is the version of the article upon submission and before peer-review. There can be important differences between the submitted version and the official published version of record.

People interested in the research are advised to contact the author for the final version of the publication, or visit the DOI to the publisher's website.

- The final author version and the galley proof are versions of the publication after peer review.

- The final published version features the final layout of the paper including the volume, issue and page numbers.

Link to publication

\footnotetext{
General rights rights.

- You may freely distribute the URL identifying the publication in the public portal. please follow below link for the End User Agreement:

www.umlib.nl/taverne-license

Take down policy

If you believe that this document breaches copyright please contact us at:

repository@maastrichtuniversity.nl

providing details and we will investigate your claim.
}

Copyright and moral rights for the publications made accessible in the public portal are retained by the authors and/or other copyright owners and it is a condition of accessing publications that users recognise and abide by the legal requirements associated with these

- Users may download and print one copy of any publication from the public portal for the purpose of private study or research.

- You may not further distribute the material or use it for any profit-making activity or commercial gain

If the publication is distributed under the terms of Article $25 \mathrm{fa}$ of the Dutch Copyright Act, indicated by the "Taverne" license above, 


\title{
Interindividual Variability and Intraindividual Reliability of Intermittent Theta Burst Stimulation-induced Neuroplasticity Mechanisms in the Healthy Brain
}

\author{
Lukas Schilberg $^{1,2}$, Teresa Schuhmann ${ }^{1,2}$, and Alexander T. Sack ${ }^{1,2}$
}

\begin{abstract}
We combined patterned TMS with EMG in several sessions of a within-subject design to assess and characterize intraindividual reliability and interindividual variability of TMSinduced neuroplasticity mechanisms in the healthy brain. Intermittent theta burst stimulation (iTBS) was applied over M1 to induce long-term potentiation-like mechanisms as assessed by changes in corticospinal excitability. Furthermore, we investigated the association between the observed iTBS effects and individual differences in prolonged measures of
\end{abstract}

\section{INTRODUCTION}

The brain's ability for dynamic structural and functional changes lies at the basis of cognitive development, including learning and memory formation. This so-called neuroplasticity refers to either cortical reorganization or changes in synaptic efficacy between neurons (Sanes \& Donoghue, 2000; Buonomano \& Merzenich, 1998; Quartz \& Sejnowski, 1997). Efforts toward a noninvasive characterization of representational neuroplasticity in humans have been made by studies applying TMS (Thickbroom, 2007; Siebner \& Rothwell, 2003). TMS allows for the induction of synaptic plasticity changes in focal regions of the cortex (Pascual-Leone et al., 1998). In combination with electrodiagnostic techniques, such as EMG, these changes can be recorded and evaluated. Combining TMS with EMG can thus be a means for the assessment and characterization of neuroplasticity processes in humans. Furthermore, by gaining insight into characteristics of neuroplasticity mechanisms in the healthy brain and by providing the means for a reliable instrument for their assessment, TMS could become a valuable tool for the development of early biomarkers of aberrant neuroplasticity processes.

Single TMS pulses (spTMS) preferably activate neuronal axons, which become electrically active and elicit action potentials that travel to the presynaptic axon terminal and release neurotransmitters into the synaptic

\footnotetext{
${ }^{1}$ Maastricht University, ${ }^{2}$ Maastricht Brain Imaging Center

(C) 2017 Massachusetts Institute of Technology
}

corticospinal excitability. Our results show that iTBS-induced measures of neuroplasticity suffer from high variability between individuals within a single assessment visit and from low reliability within individuals across two assessment visits. This indicates that both group and individual effects of iTBS on corticospinal excitability cannot be assumed to be reliable and therefore need to be interpreted with caution, at least when measured by changes in the amplitudes of motor-evoked potentials.

cleft (Huerta \& Volpe, 2009). spTMS administration at suprathreshold intensity over the primary motor cortex (M1) excites the corticospinal tract and creates a motorevoked potential (MEP) in the targeted muscle. Repetitive TMS is capable of inhibiting or facilitating cortical processes, depending on the stimulation frequency and intensity (Wassermann, 1998). Specific theta burst stimulation (TBS) protocols of TMS have been proposed to induce both long-term potentiation (LTP)- and long-term depression-like mechanisms in the targeted stimulation area (Huang, Edwards, Rounis, Bhatia, \& Rothwell, 2005). TBS-induced changes in corticospinal excitability resemble synaptic neuroplasticity mechanisms and can be assessed with spTMS-induced MEPs (Delvendahl, Jung, Kuhnke, Ziemann, \& Mall, 2012).

However, TMS-induced measures of corticospinal excitability need to be interpreted with caution, as it has been reported that the variability of MEPs is considerably large (Jung et al., 2010; Rosler, Roth, \& Magistris, 2008; Kiers, Cros, Chiappa, \& Fang, 1993) and TBS effects on corticospinal excitability can differ between individuals (Hamada, Murase, Hasan, Balaratnam, \& Rothwell, 2012). Therefore, a thorough investigation of both interindividual variability and intraindividual reliability of TBS-induced neuroplasticity measures needs to be performed to establish whether the TBS-induced systematic modulation of MEPs is indeed reliable and consistent.

Investigative approaches toward a better understanding of the reliability of TMS-induced neuroplasticity measures in the healthy brain have recently been adopted, 
and the results suggest that intraindividual reproducibility of continuous TBS (cTBS)-induced long-term depression-like mechanisms is highest at 5 min postcTBS modulation (Vernet et al., 2014). Intermittent TBS (iTBS)-induced LTP-like mechanisms have been reported to be relatively robust over two sessions and reliable at both group and individual levels (Hinder et al., 2014).

Nevertheless, we are still at the very beginning of understanding the exact mechanisms underlying neuroplasticity processes, and our tools for a meaningful assessment are not yet fully developed. To define characteristics of neuroplasticity that allow us to reliably translate information from group measures to individuals, we must find a way to reduce both inter- and intraindividual variability of the applied assessment techniques. This means that measures of neuroplasticity need to be alike within a particular cohort and that those measures need to be replicable in order for them to reliably represent measures of cortical functioning. Therefore, further indepth investigations are crucial for the development of techniques that allow for robust and generalizable characterizations of neuroplasticity mechanisms and for the meaningful assessment of possible aberrant occurrences. In the future, these measures could serve as diagnostic tools to detect abnormalities in neuroplasticity mechanisms at an early stage of their emergence, and they could help to guide therapeutic progress for affected individuals.

In this study, we investigated the reliability of iTBSinduced neuroplasticity measures over a grand time period of $60 \mathrm{~min}$, and we assessed this technique's potential to characterize and reliably measure neuroplasticity mechanisms at the individual level. In a study by Hinder et al. (2014), iTBS over M1 let to the potentiation of averaged MEP amplitudes over a time period of $36 \mathrm{~min}$ in 30 healthy young volunteers and during two separate visits. They reported no difference between the magnitude of the iTBS-induced MEP amplitude changes between the two visits and high within-subject reliability of these measures. Their study protocol included 15 spTMS-induced MEPs with a stimulation intensity of $130 \%$ individual resting motor threshold (rMT) at two baselines before iTBS and 13 post-iTBS time points. Here, we also applied iTBS to M1 in 27 participants to induce neuroplasticity-like mechanisms by changing corticospinal excitability. We examined the effectiveness of this technique by analyzing spTMS-induced group level MEP amplitude changes to baseline after iTBS administration at two separate visits. In our protocol, we applied 30 spTMS pulses with a stimulation intensity of $120 \%$ of rMT at one baseline before iTBS and at seven post-iTBS time points over a period of $60 \mathrm{~min}$. We then compared the group effects between the two iTBS visits and also between each of these visits and a third sham-iTBS visit. The latter group comparisons were performed to control for the effects of longitudinal corticospinal excitability assessment with TMS. To investigate the iTBS effects at the individual level, we examined the intraindividual reliability of these measures across visits. In addition, we investigated iTBS effects for all individuals separately to demonstrate the dimension of variability within the reported group effects. Lastly, we controlled both group and individual change to baseline measures following iTBS by individual sham-iTBS effects to verify that the baseline dependent effects of a single assessment are actually driven by the modulatory nature of iTBS and are not caused by a general trend in corticospinal excitability change during a prolonged period of assessment.

\section{METHODS}

\section{Participants}

We included 27 healthy volunteers (16 women; mean age $\pm S D=24.1 \pm 3$ years) in this study. All participants were right-handed and had a Mini-Mental State Examination score between 28 and 30. None of the participants reported having any brain-related illnesses or injuries, epilepsy, unstable medical conditions, or intake of potentially hazardous drugs before the application of TMS. The local ethics committee approved the study, and participants provided written informed consent. The research was conducted at the Department of Cognitive Neuroscience of Maastricht University in Maastricht, The Netherlands.

\section{Experimental Setup}

All participants completed two iTBS visits (Visit 1 and Visit 2) and one sham-iTBS control visit (sham-visit). The sham-visit was randomly assigned to either the first or second visit. Visit 1 and Visit 2 were on average $7.8 \pm$ $5.3(S D)$ days apart. Before the first visit, participants provided written informed consent, underwent a thorough TMS safety screening procedure, and completed the Mini-Mental State Examination.

TMS was administered with a MagPro X100 (MagVenture A/S, Farum, Denmark) stimulator and a MC-B70 figureof-eight coil. For the stimulation, the coil was placed tangentially to the scalp with the handle pointing backward at a $45^{\circ}$ angle to the midline. All delivered pulses were biphasic with the current flowing in an anteroposterior and then postero-anterior direction in the brain. The BrainVoyager neuronavigation system (Brain Innovation B.V., Maastricht, The Netherlands) was used to assure that the same cortical location of the motor hot spot was targeted throughout each session. The motor hot spot was defined as the optimal coil location on the head for maximum MEPs measured from the first dorsal interosseous (FDI) muscle of the contralateral hand. Disposable adhesive surface electrodes (Plaquette, Technomed Europe, Beek, The Netherlands) were placed in a belly-tendon montage with a ground electrode on the wrist. The EMG signals were recorded using a PowerLab 4/34 with a Bio Amp system (ADInstruments, Oxford, UK). All signals were amplified, sampled at $4 \mathrm{k} / \mathrm{sec}$, band-pass 
filtered (20-2000 Hz), digitized, and stored with LabChart (ADInstruments) for online inspection of the signal and offline analysis. To control for muscle contraction at all times throughout the measurement procedure the EMG was prepared in such a way that the resting EMG signal of the targeted FDI muscle of the right hand had a peak-to-peak amplitude of below $0.05 \mathrm{mV}$. The monitoring of that signal was continuously visible.

\section{TMS and EMG Procedure}

Participants were seated in a chair with a pillow on their lap to rest their hands on. EMG surface electrodes were placed on the participants' right hands. Neuronavigation emitters were fixated on the participants' heads (two on the forehead and one on the tip of the nose), which were then coregistered to a dummy head in BrainVoyager. The individual motor hot spot was localized by cortical mapping with single pulse TMS over M1, and the target location was marked on the dummy head to ensure consistency of stimulation location throughout the session. All participants were instructed to completely relax their hand throughout the session, and they were reminded of that whenever an EMG signal had a resting peak-to-peak amplitude higher than $0.05 \mathrm{mV}$. No TMS was administered when the participant's FDI muscle was not at rest and participants were also instructed to be completely relaxed in the period before iTBS administration. Individual rMT was determined by defining the lowest stimulation intensity needed to induce MEPs greater than $50 \mu \mathrm{V}$ peak-topeak amplitude in at least 5 of 10 consecutive trials, while the hands remained relaxed. Active motor threshold (aMT) was determined as the minimum intensity required to evoke 5 of 10 visible MEPs, while the subject maintained mild contraction of the targeted FDI muscle. Baseline MEPs were obtained by administering 30 single pulses to the target point of the left M1 at 120\% of rMT. The interval between pulses was randomized with a minimum of $7 \mathrm{sec}$. iTBS was applied to the same target point to temporarily modulate corticospinal excitability. The stimulation intensity for the iTBS protocol (20 trains of 2-sec stimulation blocks with bursts of three pulses at $50 \mathrm{~Hz}$ repeated at a 5-Hz frequency and an 8-sec delay between trains) was set at $80 \%$ of aMT (Huang et al., 2005). Following iTBS, batches of 30 neuronavigated single pulses at $120 \%$ rMT (same as baseline) were administered over the target point at 5, 10, 20, 30, 40, 50, and $60 \mathrm{~min}$ post-iTBS.

\section{Data Analysis}

EMG data were recorded with LabChart (ADInstruments). The continuous EMG signal was sampled in epochs starting 50 msec before a TMS pulse and ending $100 \mathrm{msec}$ after. Peak-to-peak MEP amplitudes were automatically defined by the software and confirmed by visual inspection. The data were preprocesses with Microsoft Excel
2010, and inferential statistical calculations were performed with IBM SPSS Statistics (Version 21).

Motor thresholds and baseline MEP values were compared between visits using the general linear model (repeated measures). The Greenhouse-Geisser correction was applied to account for violation of sphericity. The intraindividual reliability of motor thresholds and baseline MEP values over sessions were assessed using intraclass correlations. Pearson correlations were calculated between grand iTBS-induced modulations of MEPs and both rMT and aMT for Visit 1 and Visit 2 combined and for the sham-visit.

Individual MEPs were averaged for the baseline and for all time points following iTBS. To investigate the change in amplitude over time the individual ratio to baseline was calculated for each time point ([mean MEP amplitude at a particular time point (Tx) $]-[$ mean MEP amplitude at baseline $]) /($ mean amplitude at baseline). For group analyses, these individual values were averaged for all Tx. To investigate whether there was a significant positive modulation of MEPs for each visit, the overall modulation post-iTBS was compared with baseline by using one-tailed one-sample $t$ tests (two-tailed for the analysis of the shamvisit) with the test value being zero (baseline). Group effects were compared between visits using the linear mixed model analysis. Main effects were further explored using Bonferroni-adjusted post hoc comparisons. Cohen's $d$ values were calculated to determine effect sizes. All values for separate Tx analyses were outlier corrected at twice the standard deviation of the mean group value.

The reliability of iTBS effects were assessed using linear regression for an indication of the association between two variables and intraclass correlations for an indication of the reliability of the relative magnitude of each variable.

ITBS effects of both visits were controlled with shamiTBS effects by subtracting individual MEP values of all Tx and grand averages after sham-iTBS from the corresponding individual MEP values of all Tx and grand averages of Visit 1 and Visit 2. These sham-controlled values were investigated for a positive modulation with a one-tailed one-sample $t$ tests and compared between Visit 1 and Visit 2 using the mixed model analysis.

\section{RESULTS}

\section{Motor Threshold and Baseline MEPs}

Motor threshold intensities are expressed in percentage of maximum stimulator output. There was no difference in rMT (Visit 1: $43.5 \pm 1.4 \%$; Visit 2: $43.7 \pm 1.4 \%$; sham: $43.9 \pm 1.3 \% ; F(1.6,41)=0.139, p=.821$ ) or aMT (Visit 1 : $39.3 \pm 1.21 \%$; Visit 2: $39.9 \pm 1.33 \%$; sham: $40 \pm 1.35 \%$; $F(1.5,38.9)=0.583, p=.515)$ between the three visits (Figure 1A). The intraindividual reliability of motor threshold values obtained in all three visits was high for both $\operatorname{rMT}(\operatorname{ICC}(2,3)=.962, p=.000)$ and aMT (ICC $(2,3)=.955, p=.000)$ values. Pearson correlations 


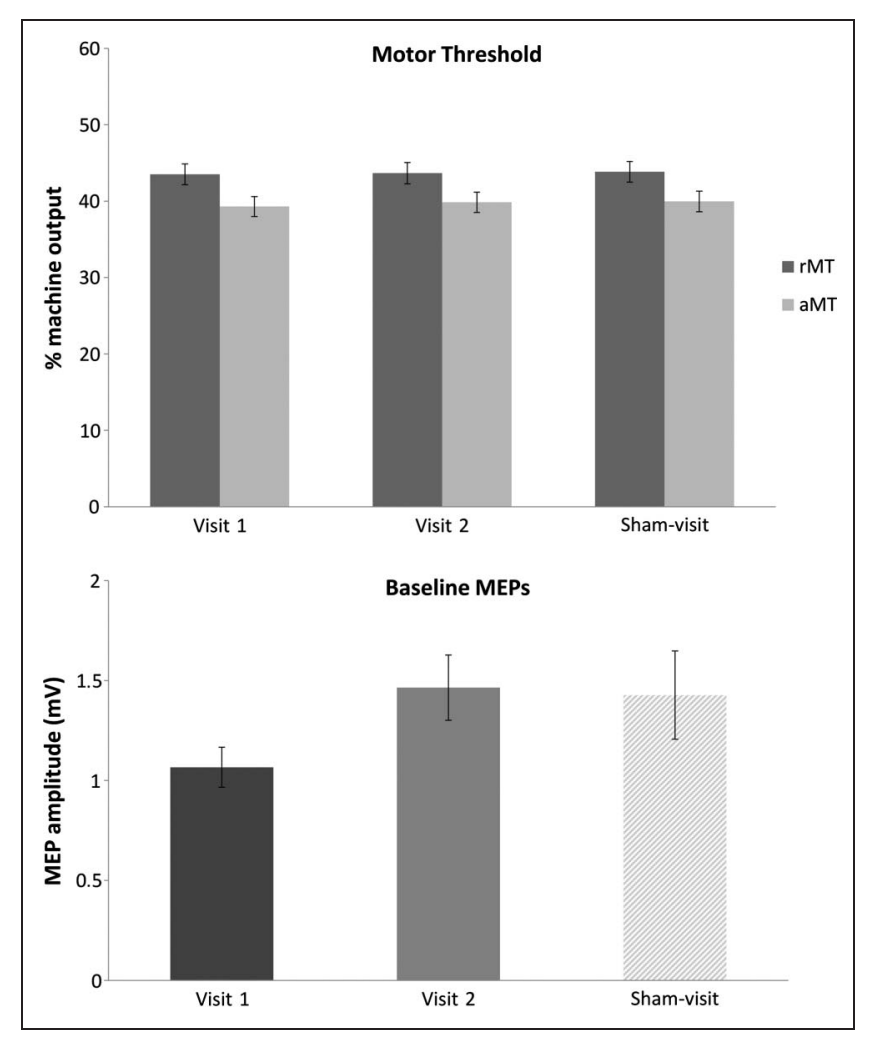

Figure 1. (A) Group-averaged machine output intensities of resting (rMT) and active (aMT) motor thresholds at both real iTBS visits (Visit 1 and Visit 2) and the sham-visit. (B) Group-averaged baseline peak-topeak amplitude of MEPs at Visit 1 , Visit 2, and sham-visit (mean $\pm S E$ ).

between the grand average of MEPs during $60 \mathrm{~min}$ (P60) post-iTBS and rMT or aMT were not significant for the two real iTBS visits (rMT: $r=.198, p=.15$; aMT: $r=$ $.134, p=.333$ ) or for the sham-visit (rMT: $r=.054, p=$ .791 ; aMT: $r=.005, p=.982$ ).
Group MEP values are expressed as the average peak-topeak amplitude of the muscle responses following spTMS of all participants. Baseline MEPs did not differ significantly between the three visits (Visit 1: $1.07 \mathrm{mV} \pm 0.1[S E]$; Visit 2: $1.46 \mathrm{mV} \pm 0.16[S E]$; sham: $1.43 \mathrm{mV} \pm 0.22[S E] ; F(1.6$, $42.5)=2.83, p=.08$; Figure $1 \mathrm{~B})$. The intraindividual reliability of baseline MEP amplitudes for all three visits was statistically significant $(\operatorname{ICC}(2,3)=.666, p=.000)$.

\section{ITBS-induced Plasticity-Group Effects}

We assessed the grand-averaged modulatory effects of iTBS on MEP amplitudes for each visit. The group modulation of corticospinal excitability (expressed as percent change to baseline) over all time points post-iTBS (60 min; P60) was significant for both Visit 1 (mean increase: $23.4 \pm 3.2[S E] ; 95 \% \mathrm{CI}:+17.1 \%$ to $+29.8 \% ; t(177)=$ $7.23, p=.000$ ) and Visit 2 (mean increase: $6.4 \pm 2.9[S E]$; $95 \%$ CI $[+.01 \%,+12 \%] ; t(179)=2.2, p=.014)$. For the sham-visit there was no significant modulation of corticospinal excitability post sham-iTBS (mean: $-2.4 \pm$ $3[S E]$; 95\% CI $[-8.2 \%,+3.4 \%] ; t(170)=-0.8, p=$ .418). Similarly, reduced to the first 35 (P35) min following iTBS a significant overall modulation of corticospinal excitability was present in Visit 1 (mean increase: $24.4 \pm$ $4.3[S E] ; 95 \% \mathrm{CI}[+16 \%,+32.8 \%] ; t(100)=7.71, p=.000)$ and Visit 2 (mean increase: $8.1 \pm 3.8[S E]$; 95\% CI [0.7\%, $+15.4 \%] ; t(102)=2.15, p=.017)$, but not for the shamvisit (mean change: $-4.6 \pm 3.7[S E]$; 95\% CI $[-11.8 \%$, $+2.5 \%] ; t(96)=-1.29, p=.21$.

The Group effect of iTBS modulation of MEP amplitudes varied significantly between visits (Figure 2). For a total of $60 \mathrm{~min}$ after iTBS modulation, the linear mixed model analysis revealed a significant main effect of Visit $(F(2,508)=18.51, p=.000)$, but no main effect of Time
Figure 2. Group-averaged percent change to baseline of peak-to-peak MEPs for post-iTBS assessment time points and grand periods of 60 (P60) and 35 (P35) min at Visit 1 , Visit 2, and sham-visit. Significant changes of $p \leq .05$ are marked with an asterisk (mean $\pm S E$ ).

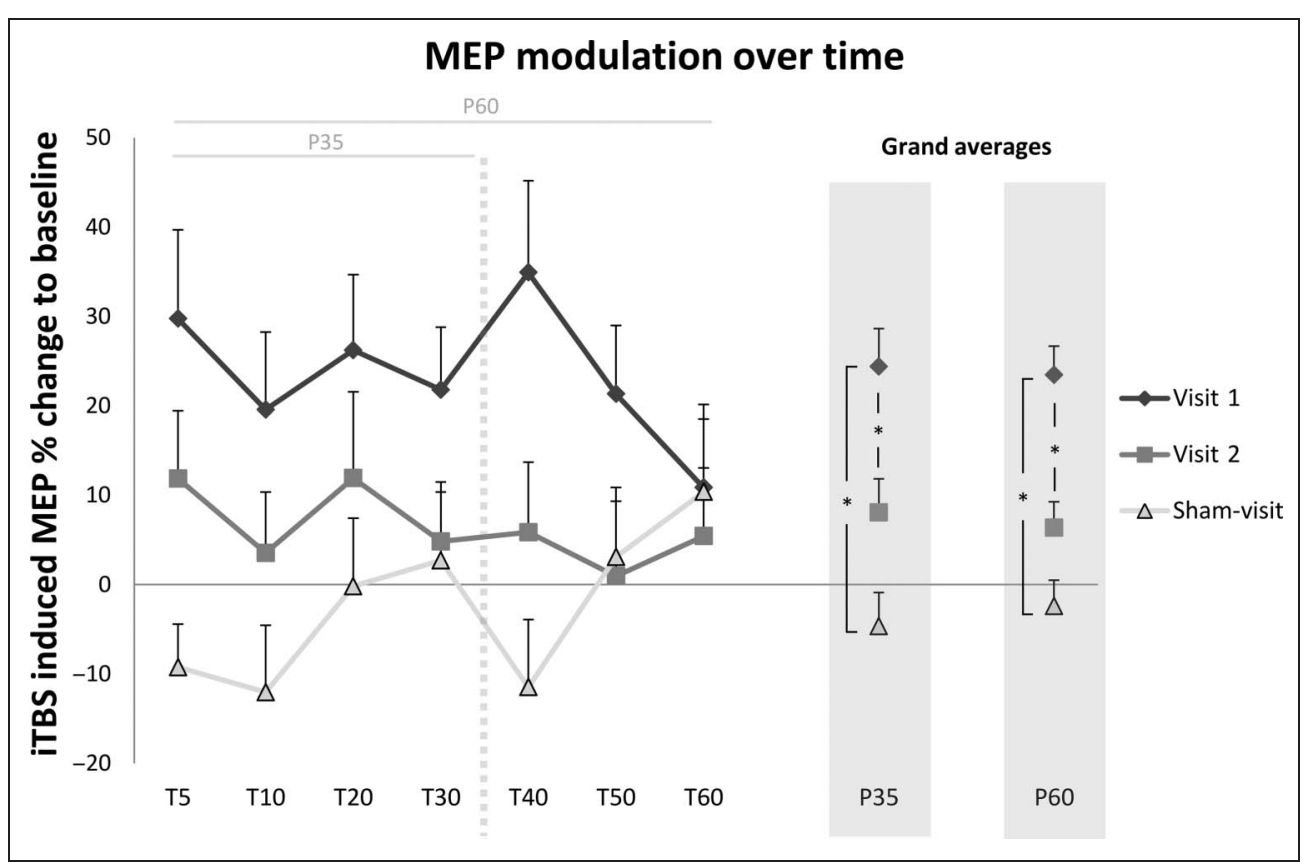




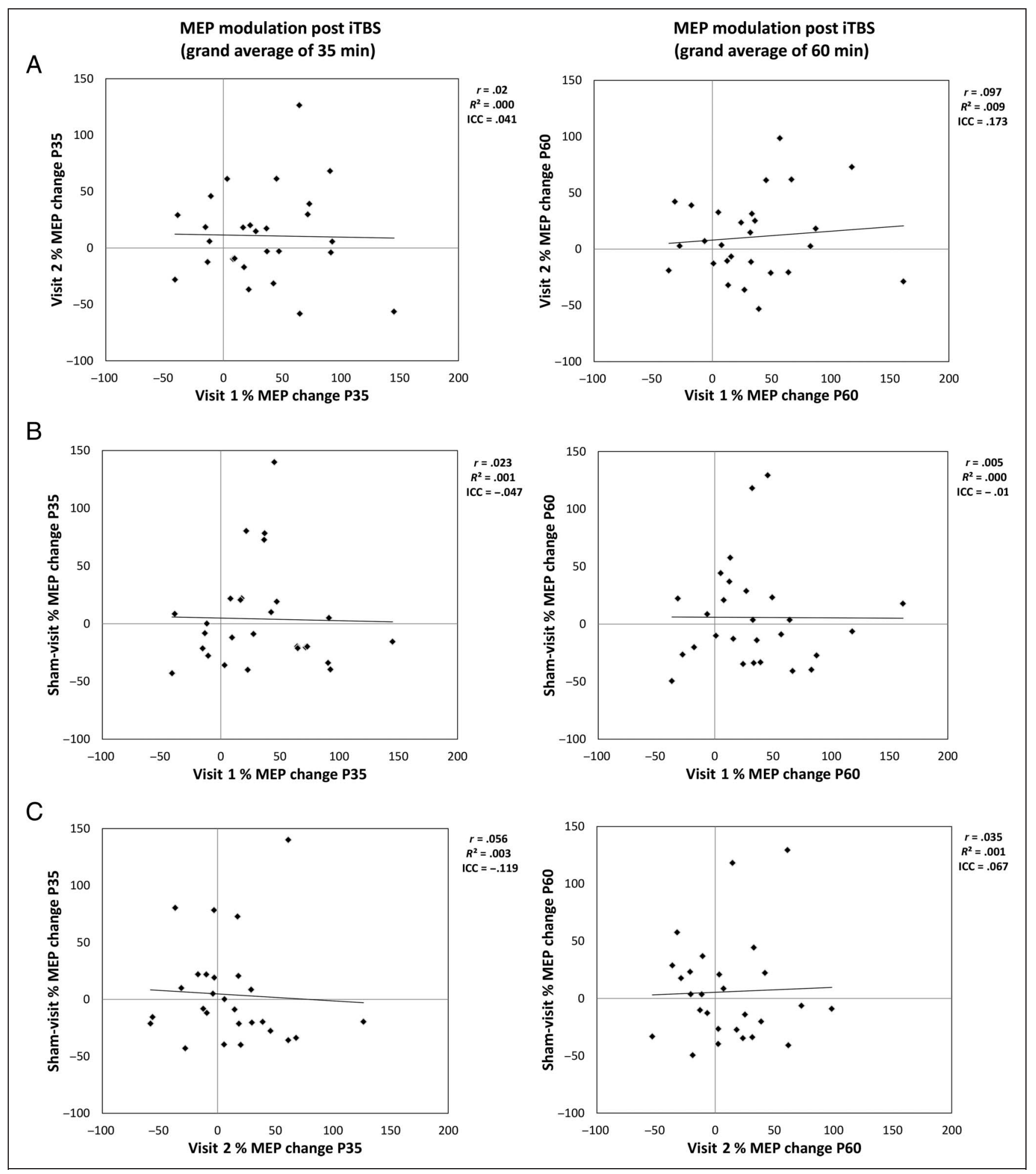

Figure 3. Individual grand-average peak-to-peak MEP amplitude changes to baseline over periods of 35 min (P35; left column) and 60 min (P60; right column) post-iTBS. Linear regressions analyses and intraclass correlations reveal no association and correlation of individual grand-average MEP modulations between (A) the two real iTBS visits (Visit 1 and Visit 2) or (B) Visit 1 and sham-visit, and (C) Visit 2 and sham-visit. All ps $<.3$

$(F(6,508)=0.35, p=.9)$ and no interaction effect $(F(12$, $508)=0.972, p=.475)$. The pairwise comparison of the three visits showed that the difference in MEP modulation was significant between Visit 1 and Visit $2(p=$ $.000 ; d=.41)$ and Visit 1 and the sham-visit $(p=.000$; $d=.63)$, but not between Visit 2 and the sham-visit $(p=$ $.132 ; d=.23)$.

Reduced to the first $35 \mathrm{~min}$ after iTBS modulation, the main effect of Visit was significant $(F(2,289)=13.46$, $p=.000)$ and the main effects of Time $(F(3,289)=0.73$, 
$p=.537)$ and the interaction effect $(F(6,289)=0.36, p=$ .905) were not significant. The pairwise comparisons revealed that there was a significant difference between Visit 1 and Visit $2(p=.010 ; d=.4)$ and Visit 1 and the sham-visit $(p=.000 ; d=.73)$. There was no significant difference between Visit 2 and the sham-visit $(p=.071$; $d=.34$ ). All $p$ values were Bonferroni-adjusted for multiple comparisons.

\section{Inter- and Intraindividual Effects}

Linear regression analyses and intraclass correlations revealed that there were no significant associations or correlations of individual MEP modulations after iTBS between Visit 1 and Visit 2, as well as between either of the two real iTBS visits and the sham-visit (Figure 3). This was the case for the overall averages during $60 \mathrm{~min}$ for Visit 1 compared with Visit $2\left(r \leq .097, R^{2} \leq .009, p \geq\right.$ .63 ; $\operatorname{ICC}(2,2) \leq .173$, ps $\geq .315)$, Visit 1 compared with the sham-visit $\left(r \leq .005, R^{2} \leq .000, p \geq .98 ; \operatorname{ICC}(2,2) \leq\right.$ $.010, p \geq .51)$, and Visit 2 compared with the sham-visit $(r \leq$ $\left..035, R^{2} \leq .001, p \geq .861 ; \operatorname{ICC}(2,2) \leq .067, p \geq .431\right)$ and during $35 \mathrm{~min}$ for Visit 1 compared with Visit $2(r \leq .020$, $\left.R^{2} \leq .000, p \geq .921 ; \operatorname{ICC}(2,2) \leq .041, p \geq .54\right)$, Visit 1 and the sham-visit $\left(r \leq .023, R^{2} \leq .001, p \geq .909 ; \operatorname{ICC}(2,2) \leq\right.$ $.047, p \geq .546)$ and Visit 2 and the sham-visit $\left(r \leq .056, R^{2} \leq\right.$ $.003, p \geq .781 ; \operatorname{ICC}(2,2) \leq .119, p \geq .611)$.

\section{ITBS Effects Compared with Sham Effects (Sham-controlled Values)}

To individualize the effects from the two iTBS visits, we took the individual MEP amplitude changes post shamiTBS as baseline for the individual MEP modulation of the two real iTBS visits. For that, we subtracted the individual average MEP amplitude change to baseline of the grand averages over 60 and $35 \mathrm{~min}$ and of each separate time point post sham-iTBS from all corresponding individual time points and grand averages of both Visit 1 and Visit 2. The grand modulation of the individual sham-controlled MEP values over 60 min were significant during Visit $1(+22.8 \pm 4.7[S E], 95 \%$ CI $[13.6 \%,+32 \%]$, $t(171)=4.876, p=.000)$, but not during Visit $2(+4.5 \pm$ $4.8[S E], 95 \%$ CI $[-4.8 \%, 13.9 \%], t(169)=0.949, p=$ .172; Figure 4). Similarly, reduced to the first $35 \mathrm{~min}$ after iTBS the grand modulation of MEPs was significant for Visit $1(+23.15 \pm 6.3[S E], 95 \%$ CI [10.8\%, 35.5\%], $t(98)=$ $3.667, p=.000)$, but not for Visit $2(+4.69 \pm 6.3[S E], 95 \%$ CI $[-7.6 \%, 17 \%], t(97)=0.748, p=.228)$. The main effect of Visit for the difference in change from the individual sham-baseline between both iTBS visits was significant over a period of $60(F(1,328)=7.224, p=.008)$ and $35 \min (F(1,189)=4.17, p=.043)$.

\section{DISCUSSION}

We here demonstrated that over a grand period of $60 \mathrm{~min}$ iTBS led to a $23.4 \%$ increase of corticospinal excitability during a first assessment visit (Visit 1), whereas shamiTBS had no modulatory effects $(-2.4 \%)$. This finding is in accordance with previous reports on iTBS-induced LTP-like effects at cortical synapses (Di Lazzaro et al., 2008; Zafar, Paulus, \& Sommer, 2008; Huang et al., 2005) and demonstrates that we successfully replicated these effects. However, most of the studies that report iTBS effects measured individual participants during a single visit only. When we repeated this first real iTBS visit with the same participants in a second assessment visit (Visit 2),
Figure 4. Group-averaged percent change to baseline of peak-to-peak MEPs for post-iTBS grand periods of 60 (P60) and 35 (P35) min at Visit 1 and Visit 2. Solid bars represent uncontrolled MEP changes, and striped bars represent sham-controlled MEP changes. For Visit 1 , the sham-controlled grand increase of corticospinal excitability following iTBS was significant for both 35 and $60 \mathrm{~min}$ after iTBS. For Visit 2, neither the sham-controlled grand increase for $35 \mathrm{~min}$, nor for $60 \mathrm{~min}$, reached statistical significance (mean $\pm S E$ ).

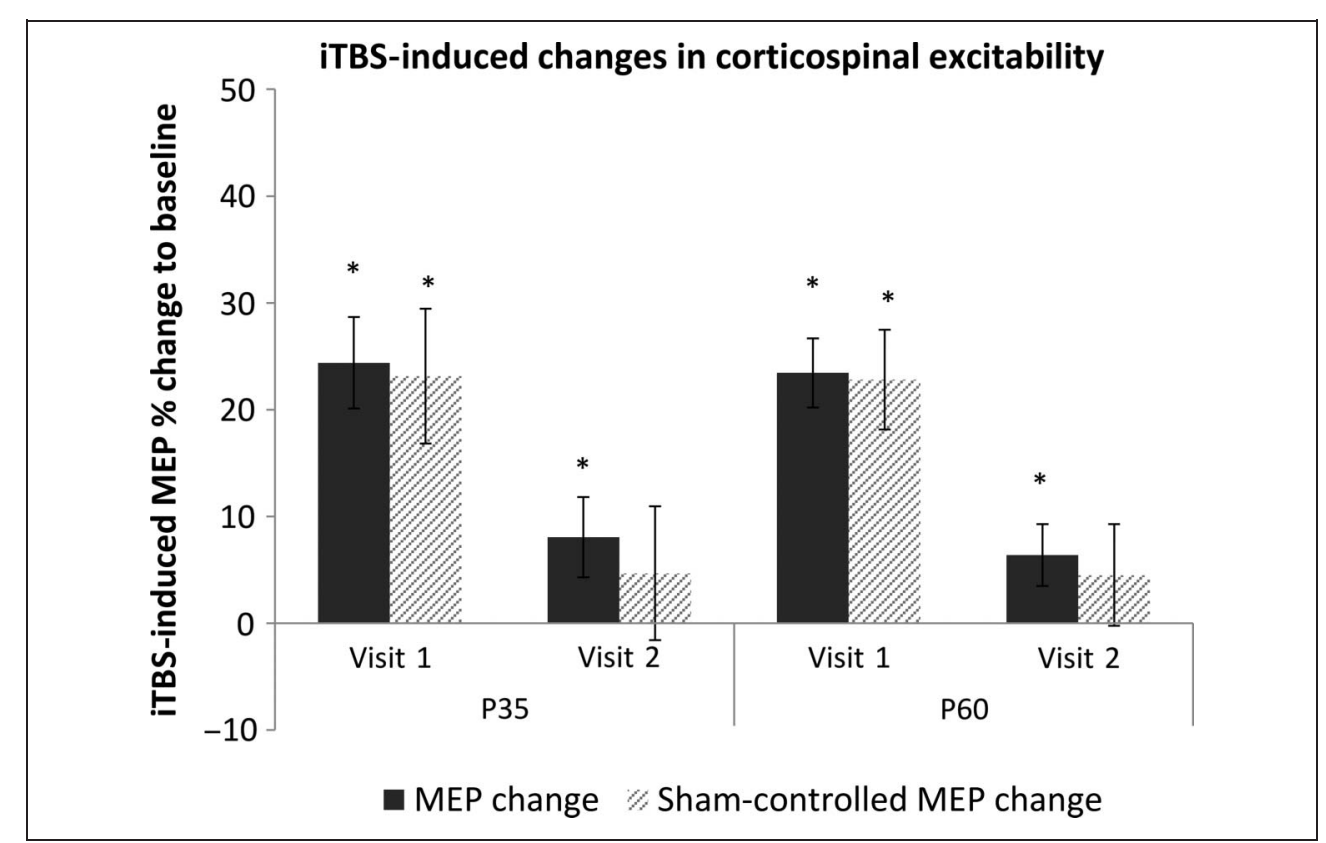


the increase of corticospinal excitability following iTBS was smaller (6.4\%). Although the facilitatory effect of iTBS still remained during the second visit, it was substantially reduced in magnitude (Figure 2). Thus, it appears that iTBS effects become weaker when testing the same participants during a second visit. On average, the interval between iTBS visits was 7.8 days, and it could be the case that the effects of iTBS habituate if two measures are repeated within a relatively short period of time. On the basis of this finding, it needs to be emphasized that the magnitude of iTBS effects cannot simply be expected to be stable across repeated assessment visits.

To match the time of interest post-iTBS of a previous study that showed reliable iTBS effects over a period of 36 min (Hinder et al., 2014), we reduced the time window for analysis from 60 to $35 \mathrm{~min}$ post-iTBS. Our results for this shorter grand period after iTBS modulation were similar to our results of the grand modulation over 60 min (Figure 2). The increase of corticospinal excitability over the time period of $35 \mathrm{~min}$ was $24.4 \%$ during the first and 8.1\% during the second assessment visit. Also for this reduced time period of interest, the facilitatory effect of iTBS remained during the second visit, but it was substantially reduced in magnitude compared with the first visit. As for the grand period of $60 \mathrm{~min}$, there was no sham-iTBS effect on corticospinal excitability for the grand period of $35 \mathrm{~min}(-4.6 \%)$. This means that even if one were to argue that iTBS effects only last for a shorter period than $60 \mathrm{~min}$, we did not find a replication of the magnitude of iTBS-induced group effects when testing the same participants repeatedly. In addition, our data showed that for both the grand effects over 60 and over $35 \mathrm{~min}$ individual measures of iTBS-induced changes in MEP amplitude did not correlate significantly between the two real stimulation visits (Visit 1 and Visit 2) nor were the individual measures reliable (Figure 3A).

The stimulation intensity was determined for each assessment visit separately based on individual corticospinal excitability on the day of that visit. On the group level, there was no difference in stimulation intensities or individual corticospinal excitability at baseline (Figure 1), and both measures were highly reliable at the individual level. Also, there were no correlations between iTBS-induced grand changes in corticospinal excitability and both rMT and aMT. Therefore, the observed intraindividual variability of iTBS-induced MEP amplitude change from baseline across the two stimulation visits was not a result of differences in stimulation intensities (120\% of rMT for spTMS; $80 \%$ of aMT for iTBS) or baseline corticospinal excitability.

To make meaningful inferences from such measures of neuroplasticity mechanisms that also hold true at the individual level, it is necessary to reach robust group effects of iTBS together with a high correlation and reliability of individual values across measurements. Unfortunately, and in disagreement with previous reports, our data suggest that the reproducibility of the magnitude of iTBS-induced group modulation of corticospinal excitability is very low. Moreover, a high correlation and reliability of individual iTBS effects between two assessments cannot be assumed based on our group results of MEP amplitude change after iTBS modulation. Instead, the low correlation between the two assessment visits shows that individual iTBS effects were not associated and low reliability measures show that also the magnitude of corticospinal excitability change was not similar for individuals. Therefore, our findings suggest that it is rather difficult to obtain meaningful information about group or individual plasticity mechanisms from a single assessment alone.

Concern about the limitation of group values of corticospinal excitability, in terms of providing valuable information about general characteristics of neuroplasticity mechanisms at the individual level, has grown recently (López-Alonso, Cheeran, Río-Rodríguez, \& Fernándezdel-Olmo, 2014). High interindividual variability of iTBS effects has been emphasized before (Nettekoven et al., 2015; Hamada et al., 2012), and our results are in accordance with this indication. We highlight in this study that besides such high interindividual variability there also appears to be high intraindividual variability of iTBS effects across visits.

So far, we have argued that because of apparently high inter- and intraindividual variability of iTBS effects it is difficult to arrive at conclusions about characteristics of neuroplasticity for individual people from the reported group values of corticospinal excitability change. Taking a closer look at the individual measures of iTBS-induced MEP amplitude modulations in this study makes it even clearer that there is great variability between individual participants during a single visit, but also within individual participants across visits. Over the grand period of $60 \mathrm{~min}$ iTBS had a significant facilitatory effect in 20 (74.1\%) of the 27 participants during the first visit and 12 participants (44.4\%) during the second visit (Figure 5). Moreover, only nine participants (33.3\%) showed significant facilitatory iTBS effects in both iTBS visits. Three participants (11.1\%) in the first visit and six participants $(22.2 \%)$ in the second visit even showed a significant inhibitory effect of iTBS. Interestingly, during the sham-visit only four participants (14.8\%) experienced no difference in MEP amplitudes compared with the baseline measures. Ten participants (37\%) experienced facilitation, and 13 participants (48.1\%) experienced inhibition in corticospinal excitability during a grand period of $60 \mathrm{~min}$ after the baseline assessment (Figure 5). Not only do these numbers demonstrate both inter- and intraindividual variability of iTBS effects, but the numbers of significant effects at the sham-visit also show that corticospinal excitability is not necessarily stable over a longer period of time (of at least 35-60 min).

To investigate individual reliability of iTBS effects in further detail, we measured the corticospinal excitability change to baseline after sham-iTBS and examined the 


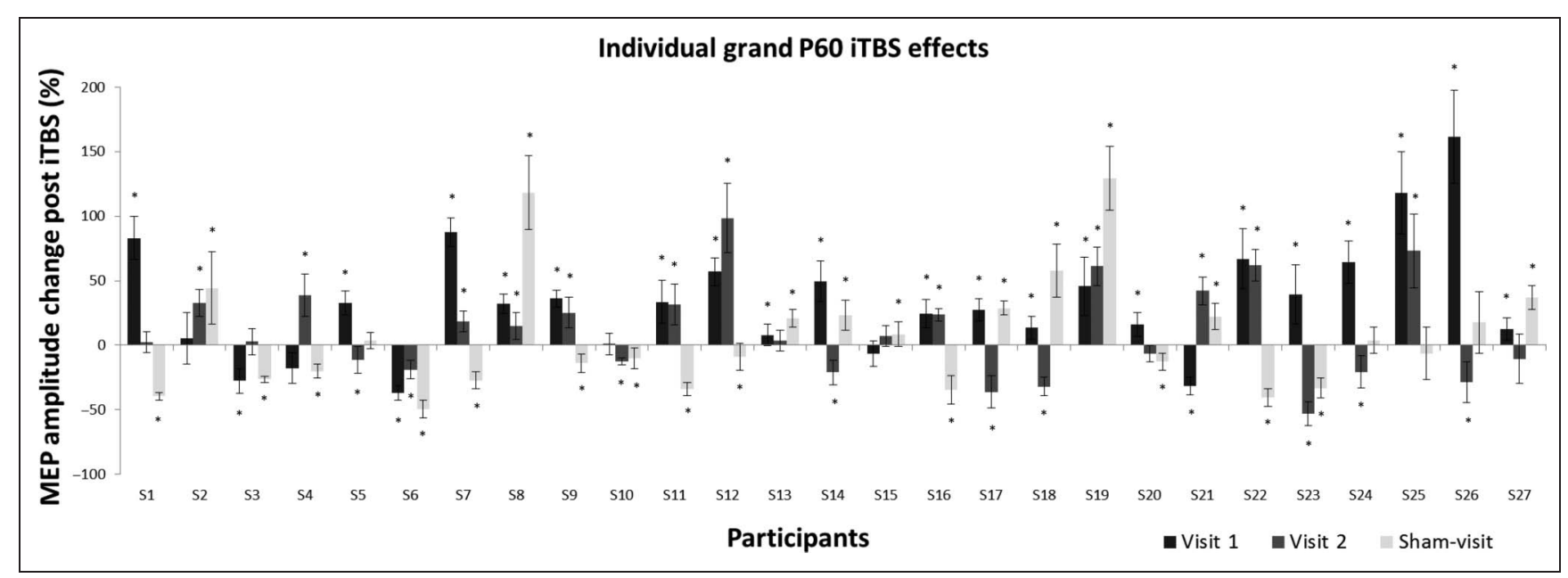

Figure 5. Average peak-to-peak MEP amplitude changes to baseline for all individual participants (S1-S27) over a grand period of 60 min (P60) post-iTBS for Visit 1 , Visit 2, and sham-visit. Significant changes to baseline of $p \leq .05$ are marked with an asterisk (mean $\pm S E$ ).

values for the difference between individual iTBS effects and individual sham-iTBS measures. By performing this sham correction of individual iTBS modulated measures we controlled for the differences in individual prolonged measures of corticospinal excitability with TMS. ShamiTBS did not lead to a significant modulation of corticospinal excitability at the group level (Figure 2), and the values for the calculated difference between individual sham-iTBS and real iTBS were similar to the group values for iTBS modulated changes in corticospinal excitability alone (Figure 4). Therefore, longitudinal MEP assessment did not influence the here presented iTBS effects during both assessment visits. It can thus be concluded that the observed effects are driven by the iTBS modulation. The pattern of a reduced iTBS effect on a second assessment day remained for the sham-controlled iTBS effects over the grand period of $60 \mathrm{~min}$. iTBS-induced facilitation of corticospinal excitability was significant for Visit 1 $(+22.8 \%)$, but no longer for Visit $2(+4.5 \%)$, and the difference between the magnitude of modulation between both visits was significant. Therefore, if individual differences of longitudinal MEP assessment are taken into account, the argument for a reduced iTBS effect on a second visit becomes even stronger. On the basis of interindividual differences in the effects of iTBS on MEP amplitudes and the finding of interindividual differences in prolonged corticospinal excitability assessments (shamvisit), it could be advantageous for the effectiveness and robustness of iTBS measures to consider incorporating individual corticospinal excitability patterns over time to the investigation of corticospinal excitability changes. Furthermore, our results show that individual measures of corticospinal excitability over a prolonged time period of up to $60 \mathrm{~min}$ (sham-visit) are not associated with the measures of iTBS during both the first and second assessment visits (Figure 3B and C). This indicates that the magnitude of iTBS effects cannot be predicted from individual prolonged corticospinal excitability patterns alone.
The above-presented individual values of changes in corticospinal excitability demonstrate that the expected facilitatory iTBS effects are variable to the extent that for some people there is no effect or even a paradoxical effect. Moreover, these values undermine our argument for high intraindividual variability of iTBS effects, which we already developed earlier in this manuscript based on our group level reliability analysis. In addition to the grand-averaged values, high intraindividual variability of MEP amplitudes at separate time points after iTBS modulation shows that a characterization of time-sensitive iTBS-induced neuroplasticity patterns is rather difficult to achieve, especially for specifically defined time points within the grand period after iTBS (see Figure 6 for individual iTBS effects at each Tx). On the basis of the variability of single pulse TMS-induced MEP amplitudes, the possible number of pulses that can be applied at a certain point in time is likely to be too small to allow for any inferences about time specific characteristics. This makes it almost impractical to develop a reliable time course of iTBS-induced neuroplasticity measures, not only at the group level, but already at the individual level.

Our results reveal that iTBS effects are not correlated or reliable between two assessments. Therefore, the intraindividual variability of iTBS-induced MEP amplitude changes from baseline is high across visits, which means that individual iTBS effects of a second assessment are not associated with individual iTBS effects of a first assessment and that the intraindividual reliability of these measures is rather low. It is important to stress that high interindividual variability of the effects of iTBS modulation on corticospinal excitability creates difficulties for the transference of group values to the individual level and that intraindividual variability of these effects across measurement visits adds complications to a reliable interpretation of both group and individual effects of a single assessment. Therefore, it seems likely that reliable characteristics of neuroplasticity cannot be derived 


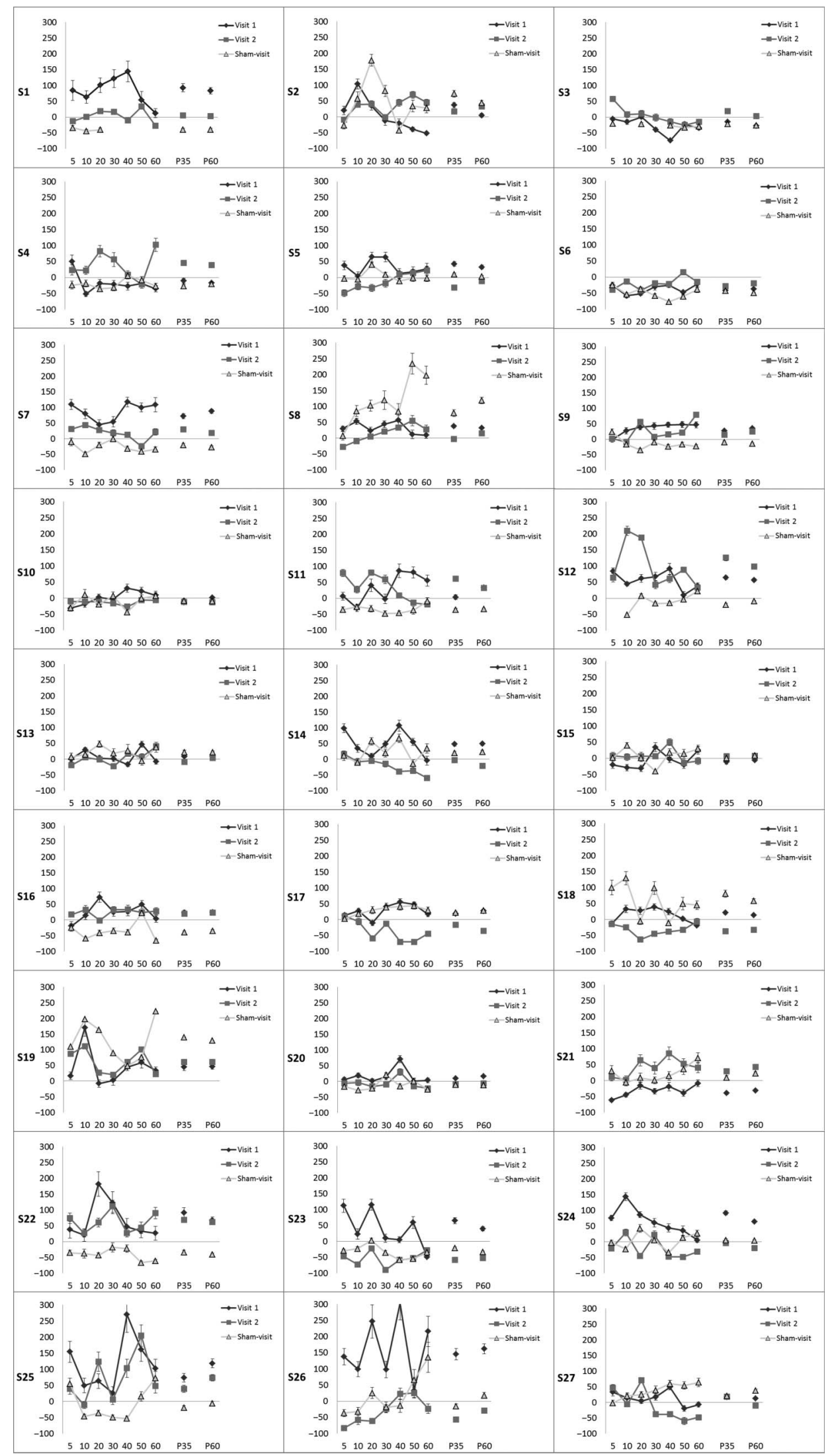

Figure 6. Average peak-to-peak MEP amplitude percent changes to baseline at all separate assessment time points and the grand periods of 35 min (P35) and $60 \mathrm{~min}$ (P60) post-iTBS of Visit 1 (diamonds), Visit 2 (squares), and sham-visit (triangles) for all participants (S1-S27; mean \pm SE). On the $y$ axes, percent changes to baseline of MEP amplitude are quantified. On the $x$ axes, the time in minutes of each separate MEP assessment and the grand averages of $35 \mathrm{~min}$ (P35) and $60 \mathrm{~min}$ (P60) are defined, (S1, S3, and S12 have missing markers because of missing data values). 
from such group values of iTBS-induced changes of corticospinal excitability.

Albeit these crucial concerns about the viability of group values of corticospinal excitability for the characterization of neuroplasticity, the grand-averaged group effects over a period of $60 \mathrm{~min}$ still demonstrate that combining iTBS with EMG has the potential to create and assess neuroplasticity in the human brain. Examining the averaged group values of iTBS-induced MEP amplitude changes alone might be a good start to demonstrate the general potential of iTBS to modulate corticospinal excitability, but these values are limited by their neglect to incorporate and control for individual differences of corticospinal excitability and vulnerable to low intraindividual reliability across measurement visits. Therefore, these measures alone seem to be inappropriate for the definition of reliable characteristics of neuroplasticity mechanisms for particular groups, as well as for individuals. To establish characteristics of neuroplasticity mechanisms that can be valuable for both particular groups and individuals and possibly even be meaningful in clinical applications, the emphasis needs to be set on the development of reliable measurement parameters of neuroplasticity mechanisms on both inter- and intraindividual levels.

It is possible that the here reported variability of iTBS effects is a result of the chosen outcome measure and not of the variability of the effect itself. The notion of a high variability of intraindividual TMS-induced MEPs has been reported (Jung et al., 2010; Rosler et al., 2008; Kiers et al., 1993), and this variability in the outcome measure might influence the measures of what is believed to represent iTBS modulation. Measuring the effect of iTBS from a single hand muscle (e.g., FDI) might just be too sensitive to individual differences in the cortical representation of that target muscle and therefore misrepresent the effect of iTBS. Therefore, it could likely be the case that MEPs are not perfectly suited for the assessment of iTBS effects. However, this does not mean that iTBS has no LTP-like effects. An alternative to MEPs as the outcome measure of choice could for instance come from neurophysiological measures assessed with EEG. The combination of online TMS and EEG could investigate the neuromodulatory effects of iTBS on the cortical level, which might be less variable on both inter- and intraindividual levels. Future studies are necessary to develop new methods to investigate neuroplasticity mechanisms. These studies will show whether iTBS has the potential to reliably investigate neuroplasticity mechanisms or whether the here reported inconsistencies of the outcome measures across and within people are actually brought about by the technique itself.

\section{Conclusions}

This study confirms that at the group level iTBS over M1 has a facilitatory effect on corticospinal excitability. How- ever, we find this group effect to be substantially weaker during a second assessment. Furthermore, there is high interindividual variability and low intraindividual reliability of the observed iTBS-induced neuroplasticity measures. High variabilities of these measures, both between and within people, create great challenges for the interpretation of group iTBS effects on corticospinal excitability, and they make it difficult to define general characteristics of neuroplasticity mechanisms that are reliably transferable to the individual level. Moreover, our findings indicate that group results of iTBS modulation that are based on a single assessment need to be interpreted with caution before conclusions can be drawn about general mechanisms of neuroplasticity. It cannot be assumed that single assessment group measures of iTBS-induced changes in corticospinal excitability are representative of group-specific characteristics of neuroplasticity mechanisms. Moreover, the relevance of these measures for the interpretation of individual neuroplasticity measures is questionable, at least when assessed with MEPs from a single hand muscle. Further research is required to improve the existing techniques and establish application parameters that reliably measure neuroplasticity with both low inter- and intraindividual variability to arrive at generalizable characteristics of neuroplasticity that are reliably measurable in individuals.

\section{Acknowledgments}

This work was supported by a grant from the Netherlands Organization for Scientific Research (NWO; HCMI 10-19). We thank Melloney Wijenberg for her help with participant recruitment and data collection. Design of the experiment: L. S., T. S., and A. T. S. Data collection: L. S. Analysis and interpretation of the data: L. S., T. S., and A. T. S. Writing of the manuscript: L. S., T. S., and A. T. S.

Reprint requests should be sent to Lukas Schilberg or Prof. Alexander T. Sack, Faculty of Psychology and Neuroscience, Department of Cognitive Neuroscience, Oxfordlaan 55, Maastricht 6229 EV, The Netherlands, or via e-mail: lukas.schilberg@ maastrichtuniversity.nl, a.sack@maastrichtuniversity.nl.

\section{REFERENCES}

Buonomano, D. V., \& Merzenich, M. M. (1998). Cortical plasticity: From synapses to maps. Annual Review of Neuroscience, 21, 149-186.

Delvendahl, I., Jung, N. H., Kuhnke, N. G., Ziemann, U., \& Mall, V. (2012). Plasticity of motor threshold and motor-evoked potential amplitude-A model of intrinsic and synaptic plasticity in human motor cortex? Brain Stimulation, 5, 586-593.

Di Lazzaro, V., Pilato, F., Dileone, M., Profice, P., Oliviero, A., Mazzone, P., et al. (2008). The physiological basis of the effects of intermittent theta burst stimulation of the human motor cortex. Journal of Physiology, 586, 3871-3879.

Hamada, M., Murase, N., Hasan, A., Balaratnam, M., \& Rothwell, J. C. (2012). The role of interneuron networks in driving human motor cortical plasticity. Cerebral Cortex, 23, 1593-1605.

Hinder, M. R., Goss, E. L., Fujiyama, H., Canty, A. J., Garry, M. I., Rodger, J., et al. (2014). Inter- and intraindividual variability following intermittent theta burst stimulation: Implications for rehabilitation and recovery. Brain Stimulation, 7, 365-371. 
Huang, Y. Z., Edwards, M. J., Rounis, E., Bhatia, K. P., \& Rothwell, J. C. (2005). Theta burst stimulation of the human motor cortex. Neuron, 45, 201-206.

Huerta, P. T., \& Volpe, B. T. (2009). Transcranial magnetic stimulation, synaptic plasticity and network oscillations. Journal of Neuroengineering and Rehabilitation, 6, 7.

Jung, N. H., Delvendahl, I., Kuhnke, N. G., Hauschke, D., Stolle, S., \& Mall, V. (2010). Navigated transcranial magnetic stimulation does not decrease the variability of motor-evoked potentials. Brain Stimulation, 3, 87-94.

Kiers, L., Cros, D., Chiappa, K. H., \& Fang, J. (1993). Variability of motor potentials evoked by transcranial magnetic stimulation. Electroencephalography and Clinical Neurophysiology, 89, 415-423.

López-Alonso, V., Cheeran, B., Río-Rodríguez, D., \& Fernándezdel-Olmo, M. (2014). Inter-individual variability in response to non-invasive brain stimulation paradigms. Brain Stimulation, 7, 372-380.

Nettekoven, C., Volz, L. J., Leimbach, M., Pool, E. M., Rehme, A. K., Eickhoff, S. B., et al. (2015). Inter-individual variability in cortical excitability and motor network connectivity following multiple blocks of rTMS. Neuroimage, 118, 209-218.

Pascual-Leone, A., Freitas, C., Oberman, L., Horvath, J. C., Halko, M., Eldaief, M., et al. (2011). Characterizing brain cortical plasticity and network dynamics across the age-span in health and disease with TMS-EEG and TMS-fMRI. Brain Topography, 24, 302-315.

Quartz, S. R., \& Sejnowski, T. J. (1997). The neural basis of cognitive development: A constructivist manifesto.
Behavioral and Brain Sciences, 20, 537-556; discussion 556-596.

Rosler, K. M., Roth, D. M., \& Magistris, M. R. (2008). Trial-to-trial size variability of motor-evoked potentials. A study using the triple stimulation technique. Experimental Brain Research, 187, 51-59.

Sanes, J. N., \& Donoghue, J. P. (2000). Plasticity and primary motor cortex. Annual Review of Neuroscience, 23, 393-415.

Siebner, H. R., \& Rothwell, J. (2003). Transcranial magnetic stimulation: New insights into representational cortical plasticity. Experimental Brain Research, 148, 1-16.

Thickbroom, G. W. (2007). Transcranial magnetic stimulation and synaptic plasticity: Experimental framework and human models. Experimental Brain Research, 180, 583-593.

Vernet, M., Bashir, S., Yoo, W. K., Oberman, L., Mizrahi, I., Ifert-Miller, F., et al. (2014). Reproducibility of the effects of theta burst stimulation on motor cortical plasticity in healthy participants. Clinical Neurophysiology, 125, 320-326.

Wassermann, E. M. (1998). Risk and safety of repetitive transcranial magnetic stimulation: Report and suggested guidelines from the International Workshop on the Safety of Repetitive Transcranial Magnetic Stimulation, June 5-7, 1996. Electroencephalography and Clinical Neurophysiology, 108, 1-16.

Zafar, N., Paulus, W., \& Sommer, M. (2008). Comparative assessment of best conventional with best theta burst repetitive transcranial magnetic stimulation protocols on human motor cortex excitability. Clinical Neurophysiology, 119, 1393-1399. 